\title{
Re: Effects of Recurrent Urinary Tract Infections on Graft and Patient Outcomes After Kidney Transplantation
}

\author{
Britt NS', Hagopian JC',2, Brennan $\mathrm{DC}^{2}$, Pottebaum $\mathrm{AA}^{1}$, Santos $\mathrm{CAO}^{3}$, Gharabagi $\mathrm{A}^{2}$, Horwedel $\mathrm{TA}^{1,2}$ \\ 'Barnes-Jewish Hospital, Clinic of Pharmacy, St. Louis, USA \\ ${ }^{2}$ Washington University Faculty of Medicine, Department of Nephrology, St. Louis, USA \\ ${ }^{3}$ Rush University Medical Center, Department of Internal Medicine, Division of Infectious Diseases, Chicago, USA \\ Nephrol Dial Transplant 2017;32:1758-1766. doi: 10.1093/ndt/gfx237.
}

\section{EDITORIAL COMMENT}

In this retrospective single center study at a large-volume center, the authors have investigated association between post kidney transplant (KT) urinary tract infections (UTIs) and graft and patient outcomes, with a focus on recurrent (R)-UTIs ( $\geq 2$ UTIs in any 6-month period or $\geq 3$ UTIs in any 12-month period). Of 2469 patients included, 1835 (74.3\%) had no UTI, 465 (18.8\%) had nonrecurrent (NR)-UTI (urine sample containing $>10^{5}$ bacterial colony-forming units/mL) and 169 (6.8\%) had R-UTI. The authors have found that patients with R-UTI had significantly worse graft function and graft survival compared with both those with no UTI as well as NR-UTI. R-UTI, but not NR-UTI, was associated with poorer patient survival compared with no UTI. Therefore, the association between UTI and worse patient outcomes in the KT population appears to be driven by recurrent cases. R-UTIs were more likely to be caused by multi drug resistant organisms, posing unique treatment challenges. Also, some risk factors, which have not been previously described, were found in this study for R-UTIs; increased age, African-American race and deceased donor status were also reported besides female gender, diabetes mellitus, ureteric stenting, re-transplantation, lack of antimicrobial prophylaxis and prolonged urinary catheterization which have been linked to R-UTI in the literature.

Yarkın Kamil Yakupoğlu, MD

बCopyright 2018 by the Association of Urological Surgery / Journal of Urological Surgery published by Galenos Publishing House. 